\title{
Easy Rule for Tuning Organs and Harmoniums in Equal Temperament
}

Author(s): Alexander J. Ellis

Source: The Musical Times and Singing Class Circular, Vol. 20, No. 440 (Oct. 1, 1879), pp. 520-521

Published by: Musical Times Publications Ltd.

Stable URL: http://www.jstor.org/stable/3356776

Accessed: 30-05-2016 18:56 UTC

\footnotetext{
Your use of the JSTOR archive indicates your acceptance of the Terms \& Conditions of Use, available at

http://about.jstor.org/terms
}

JSTOR is a not-for-profit service that helps scholars, researchers, and students discover, use, and build upon a wide range of content in a trusted digital archive. We use information technology and tools to increase productivity and facilitate new forms of scholarship. For more information about JSTOR, please contact support@jstor.org.

Musical Times Publications Ltd. is collaborating with JSTOR to digitize, preserve and extend access to The Musical Times and Singing Class Circular 
effect. After repeating much that has been already quoted, the master went on: "If I solicit it (the directorship) I am bound to accept the place as they choose to give it, and to comply with their conditions as to salary, duties, \&c., though I do not as yet even know what these are. In the second place, the reason they gave you why I should write seems to me neither a true nor a straightforward one. They say they wish to be certain I will accept it, and that, on this account, I must enrol myself among the candidates; but when they offered it to me three years ago, Lichstenstein said they did so to ascertain if $\mathrm{I}$ would take it, and begged me to give a distinct answer on the point. At that time I said 'Yes,' that I was willing to carry it on, along with Rungenhagen. I am not sure that I should think the same now; but as I said so then, I cannot draw back, and must keep my word. It is not necessary to repeat my assent, for as I once gave it so it must remain; still less can I do so when I should have to offer myself to them for the post they once offered to me. If they were disposed to adhere to their former offer they would not require me to take a step which they took themselves three years ago; on the contrary, they would remember the assent I then gave, for they must know I am incapable of breaking a promise. A confirmation of my former promise is, therefore, quite unnecessary, and if they intend to appoint another to the situation, my letter would not prevent their doing so." The sequel, as told by Devrient, proves that Mendelssohn fils in London had a much clearer perception of the actual situation than Mendelssohn pève in Berlin. Rungenhagen, who long acted as Zelter's deputy, had many friends, and a strong body of members were of opinion that, albeit lacking Mendelssohn's brilliant talents, he could not be passed over. The matter was debated for six months, and then an effort was made to compromise it by appointing a double conductorship, with Rungenhagen as the first of two equals, Mendelssohn all the time declining to appear as an avowed candidate. Hot and stormy were the discussions that arose, some members declaring that "well-born" ladies and gentlemen were not to be dictated to by so young a man, and others protesting that, the Academy being a Christian institution, it was absurd to put a Jewish lad at the head. Nevertheless, the joint conductorship would have been established but for Rungenhagen himself, who would have all or none, and demanded that the question should be put to the vote. To the vote it was accordingly put in January, 1833 , with the following result: Rungen. hagen, I48; Mendelssohn, 88; Grell, 4. Devrient, Mendelssohn's foremost champion, was excessively mortified at this defeat, while the composer's. family took it so much to heart that they seceded in a body from the Academy. As for the master himself he preserved entire calmness, and whatever pain the rebuff entailed probably arose from a feeling that he should have adhered strictly to first impulse, and not even have sanctioned a candidature promoted by well-meaning though mistaken friends against his own better judgment.

$$
\text { (To be continued.) }
$$

\section{EASY RULE FOR TUNING ORGANS AND} HARMONIUMS IN EQUAL TEMPERAMENT. By Alexander J. Ellis, F.R.S.

THE following rule is intended to supply an easy mechanical method of tuning in Equal Temperament without sensible error. It was originally given in my translation of Helmholtz's "Sensations of Tone" (p. 785), and was mentioned with approval by Mr. Bosanquet in his work on "Temperament" (p. 5).
It is now presented in a greatly improved form. It is especially adapted to organs and harmoniums, as the pianoforte tones are not sustained enough for counting beats with certainty; but it will be found valuable even to pianoforte-tuners. It is suitable for all pitches from $\mathrm{C}_{500}$, which is as flat as our flattest organs in cold weather, up to at least $\mathrm{C}_{587} 7^{\circ}$, which is the enormously high pitch of the St. James's Church Organ at Hamburg. The examples given are, for English band-pitch, $\mathrm{C}_{540}$, and French pitch, A $435^{\circ} 4$ (not A 435 , as appears from measurements of the diapason normal itself at the Musée du Conservatoire at Paris, taken in 1878 ).

Apparatus.-Besides the tuning tools, either a seconds watch or else two little pendulums swinging twice and three times in the second. These can be made with quite sufficient accuracy by passing a thin thread or piece of sewing silk through a heavy button and tying it in a complete loop, so that one end can be passed over a pencil or smooth wire or nail. The whole length of the double loop, or swinging length of the pendulum, should be 9 inches $12 \frac{1}{2}$ sixteenths for the half-second, and 4 inches $5^{\frac{1}{2}}$ sixteenths for the third of a second pendulum. But any clockmaker would make them of metal with a bob that screws, and then they could be carefully adjusted to accurate time.

Rule.-For Organs and Harmoniums.-Take the bearings on the one-foot octave only, from $c^{\prime \prime}$ on the third space of the treble to the $b^{\prime \prime}$ above.

I. For High Pitches. - Take the $c^{\prime \prime}$ to any pitch required, or leave it as it stands. Tune by fifths up and fourths down, making the note to be tuned at first tolerably correct for perfect intervals (that is, without sensible beats), and then flattening it in each case, so as to make the fifths beat twice and the fourths beat three times in a second. See Scheme No. I.

2. For Medium Pitches, near to French Pitch.-Begin with $a^{\prime \prime}$, and tune by fifths down and fourths up till you reach $c^{\prime \prime}$, always bringing the note to be tuned nearly correct for perfect intervals (that is, without sensible beats), and then sharpening it in each case, so as to make the fifths beat twice and the fourths three times in a second. Then begin again at $a^{\prime \prime}$, and proceed as in the first case. See Scheme No. 2.

3. For very Low Pitches indeed, as for very Flat Organs in Winter.-Begin with $a^{\prime \prime}$ and tune as in the last case by a fifth $d o w n$ to $d^{\prime \prime}$ and then by a fourth up to $g^{\prime \prime}$, sharpening the note to be tuned after making it nearly perfect. Then begin again with $a^{\prime \prime}$, and proceed as in the first case, but continue the process to $b^{\prime \prime}=c^{\prime \prime \prime}$. See Scheme No. 3 .

The remaining octaves will then be tuned from the bearings in the usual way, and the result may be verified by beats. But it must be remembered that the two beats and three beats of the fifths and fourths which occur in one second in the one-foot octave occur in two seconds in the two-foot octave, in four seconds in the four-foot octave, and in eight seconds in the eight-foot octave. Such beats are too slow to tune by, but serve well as a verification.

\section{SCHEMES FOR TUNING.}

Notes alveady tuned, white. Notes to be tuned, black. Number of beats to be heard in a second written below each interval.

\section{No. I. For High Pitches.}

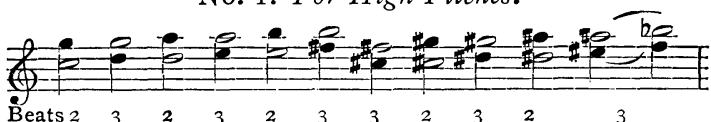

Bring the black note to form nearly a perfect fifth or fourth, as the case may be, with the white, and then flatten it. 
No. 2. For Medium Pitches, near French Pitch.

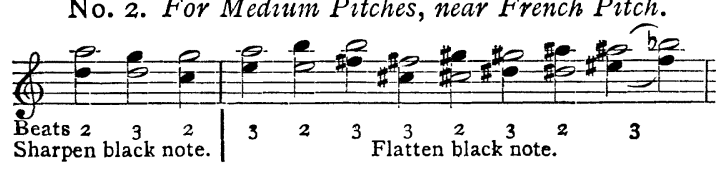

No. 3. For extremely Low Pitches.

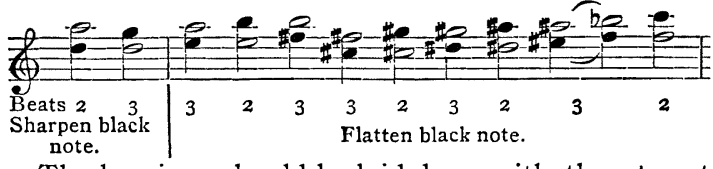

The bearings should be laid down with the utmost accuracy, the beats counted, if possible, for ten seconds, the bellows being well filled and not repumped during the count of any one set of beats; each set of beats should be counted three or four times over, and the work should be rigidly revised, when the bearings have been completely tuned, by recounting the beats, if possible, by some other person and not the tuner. This gives a mechanical proof of the work not otherwise possible. Not till then should the rest of the instrument be tuned. It is important for the organ that the temperature should remain constant while the bearings are taken, but the actual temperature is indifterent, and the octaves from these bearings may be taken at any other temperature. To prevent inequalities of temperature while tuning the bearings, great care should be taken not to heat the pipes by touching them, or by bringing the body too close, and no pipe after tuning should be removed from its place.

When great accuracy is required it is important that the reeds of harmoniums should be allowed to settle for a day or two after the bearings have been taken, and then be re-proved by counting the beats, before the other octaves are tuned from them.

For Pianofortes.-The beats last so short a time that the rule requires modification. The bearings should be taken exactly one octave lower than in the schemes, that is, in the two-foot octave. The fifths should beat four times and the fourths six times in four seconds, the utmost time for which they can be counted with accuracy. Hence it is not possible to secure such perfect evenness of temperament on the pianoforte as on the organ and harmonium, but the error is not so easily perceived, and the resulting equality is much greater than can be secured by mere estimation of ear. Of course the bearings must be taken on single strings, the others being damped, and great care should be subsequently taken with the unisons. If two or three pianos are being tuned in the same room at the same time, great practice is necessary to distinguish the beats.

Precaution in counting Beats.-It is usual in counting to begin with one. If we begin to count one at the beginning of the first second, two at the beginning of the second second, and so on, we shall have said six at the beginning of the sixth second, which is also at the end of the fifth second; that is, we shall have counted for only five entive seconds, and the beats will have been five (not six) in five seconds-that is, one in a single second. We must, therefore, always throw off one from our count for beginning with one in place of nought. Great mistakes are often made in counting beats from neglecting this precaution. The tuner should practise counting the ticks of a watch, which will be generally four or three in one second, or five in two seconds. Clocks tick one, two, and four times in a second.

\section{Examples.}

The columns Correct give the pitches (that is, the numbers of double vibrations in one second) which each note in the scale ought to have, if it were tuned in mathematically equal temperament, for Band Pitch, $c^{\prime \prime} 540$, and French Pitch, $a^{\prime \prime} 870 \cdot 80$. They are all continued to two places of decimals, so that if the point is disregarded the figures show the number of double vibrations in a hundred seconds.

The columns By Rule give the pitches of each note as they would have if tuned with mathematical accuracy by my rule. It will be seen that the two sets of pitches-Correct and By Rule-never differ by two-tenths, that is, one-fifth of a vibration, or one vibration in five seconds, a difference which Professor W. Preyer, of Jena, has proved to be insensible in any part of the great scale.

The columns headed Error give the nearest number of thousandths of an equal semitone by which a note, as tuned by rule, is sharper $(\sharp)$ or flatter (b) than it would be if tuned in perfectly equal temperament. It will be seen that the largest error (marked $4 \mathrm{D}$ ) is less than the two-hundred and fiftieth part of a semitone too flat, and is absolutely inappreciable by the finest ear.

The notes should have been doubly accented as belonging to the one-foot octave, but the accents are omitted for convenience.

\begin{tabular}{|c|c|c|c|c|c|c|c|}
\hline \multicolumn{4}{|c|}{ BAND PITch. } & \multicolumn{4}{|c|}{ French Pitch. } \\
\hline Notes. & Correct. & By Rule & Error. & Notes. & Correct. & By Rule & Error. \\
\hline c & $540^{\circ} 00$ & $540^{\circ} \mathrm{Oo}$ & 0 & c & 517.78 & 517.93 & 0 \\
\hline$c$ & $572 \mathrm{II}$ & $572^{\circ} 08$ & I & c & 548.58 & 548.55 & o \\
\hline$d^{\prime \prime}$ & 6.6 .12 & 606.00 & $4 ?$ & $d^{\prime \prime}$ & $50 \mathrm{r} 20$ & 58120 & $o_{1}$ \\
\hline $\mathrm{d}$ & $642 \cdot 17$ & 642.09 & $2 b$ & $\mathrm{~d}$ & 61576 & 615.62 & $4^{b}$ \\
\hline $\mathrm{e}^{\prime \prime}$ & 680.30 & $680^{\circ} 25$ & 20 & $e^{*}$ & 652.37 & 652.35 & 0 \\
\hline f & $720^{\prime} 78$ & 720.85 & $I=$ & $\mathrm{f}$ & $69 \mathrm{r} \cdot 17$ & 691.07 & $2 b$ \\
\hline $\mathrm{fH}$ & 763.67 & 763.78 & 2 & $f$ & $732 \cdot 27$ & $7322^{\circ}$ & 3 \\
\hline g & $809^{\circ} 07$ & $809^{\circ} 00$ & $2 \tilde{D}$ & g & $775^{\circ} 80$ & 77593 & 3 \\
\hline$g$ & $857^{\circ} \cdot 20$ & $85 \% \cdot 12$ & 21 & $g=$ & $82 \mathrm{r} 94$ & 821.83 & $2 b$ \\
\hline a & $908 \cdot 17$ & 908.00 & 36 & $a^{\circ}$ & 870.80 & 870.80 & 0 \\
\hline a* & $962 \cdot 16$ & $962 \cdot 13$ & I b & a & $922 \cdot 60$ & 922.43 & 30 \\
\hline$b^{\prime \prime}$ & I019.36 & I019.37 & 0 & $b^{\prime \prime}$ & $977^{\circ} 44$ & 97753 & $2 b$ \\
\hline
\end{tabular}

If we calculate the beats in one second of the fifths and fourths of mathematically equal temperament in these two cases from the numbers given in the columns called Correct, in order to contrast them with the constant two and three beats of the fifths and fourths when tuning By Rule, we find them as follows :-

\begin{tabular}{|c|c|c|c|}
\hline \multicolumn{2}{|c|}{ Band Pitch. } & \multicolumn{2}{|c|}{ French Pitch. } \\
\hline Fifths. & Beats. & Fifths. & Beats. \\
\hline $\begin{array}{ll}c & g \\
c & g \\
d & a \\
d & a \\
e & b\end{array}$ & $\begin{array}{l}\mathbf{I} \cdot 86 \\
\mathbf{r} \cdot 93 \\
2 \cdot 02 \\
2 \cdot 19 \\
2 \cdot 18\end{array}$ & $\begin{array}{ll}c & g \\
c & g \\
d & a \\
d & a \\
e & b\end{array}$ & $\begin{array}{l}I \cdot 74 \\
I \cdot 86 \\
2 \cdot 00 \\
2 \cdot 08 \\
2 \cdot 23\end{array}$ \\
\hline Fourths. & Beats. & Fourths. & Beats. \\
\hline $\begin{array}{ll}c & f \\
c \neq & f \\
d & g \\
d & g \\
e & a \\
f & b b \\
f & b\end{array}$ & $\begin{array}{l}2.34 \\
2.57 \\
2.73 \\
2.92 \\
3.31 \\
3.36 \\
3.40\end{array}$ & $\begin{array}{ll}c & f \\
c \neq & f \neq \\
d & g \\
d & g \\
\text { e } & a \\
f & b b \\
f & b\end{array}$ & $\begin{array}{l}2 \cdot 39 \\
2 \cdot 39 \\
2 \cdot 60 \\
2 \cdot 78 \\
2 \cdot 92 \\
3 \cdot 12 \\
3 \cdot 24\end{array}$ \\
\hline
\end{tabular}

If we take the average of these beats, excluding the fourth $c f$, which is not tuned by the rule, we find:-

\begin{tabular}{c|c|c|c}
\hline Mean of Beats & Band Pitch. & French Pitch. & Mean of both. \\
\cline { 1 - 2 } & $\begin{array}{c}\text { Fifths } \\
\text { Fourths }\end{array}$ & $\begin{array}{c}\mathbf{1} \cdot 98 \\
2 \cdot 84\end{array}$ & $\begin{array}{c}2 \cdot 005 \\
2 \cdot 945\end{array}$ \\
\hline
\end{tabular}

It appears, therefore, that the rule gives as nearly as possible the mean amount of beats in, all cases, being sometimes too much and sometimes too little by an extremely small amount. It was upon such observations that the rule was originally founded. 